\title{
SHORT WAVE INSTABILITIES RESULTING FROM MEMORY SLIP
}

By

Michael Renardy

IMA Preprint Series \# 556

August 1989 


\section{Short wave instabilities}

resulting from memory slip

Michael Renardy

Department of Mathematics and ICAM

Virginia Tech

Blacksburg, VA 24061-0123 


\section{Abstract}

It is shown that the equations governing the flow of an upper convected Maxwell fluid can become ill-posed if the no slip condition at the wall is replaced by a law of "memory slip" which relates the slip velocity to the history of the shear stress.

\section{Introduction}

Recent experiments by Ramamurthy [1] have demonstrated that the onset of melt fracture is associated with the development of slip at the wall. A sequence of several different instabilities can be observed, see e.g. Kalika and Denn [2]. After slip begins, short lengthscale irregularities develop which manifest themselves as loss of gloss and "sharkskin". These irregularities seem to be more or less confined to the boundary, while the overall flow appears to be steady. At higher shear rates, sharkskin regions with slow slip alternate with glossy regions of rapid slip (stick-slip regime). As the shear rate is increased still further, the extrudate again becomes completely glossy, and wavy distortions appear.

The mechanism by which slip occurs and how slip leads to the observed instabilities are not understood. Twenty years ago Pearson and Petrie [3] investigated the stability of shear flows of various fluids when the slip velocity at the wall is a given function of the shear stress: $u=f(\tau)$. As one might expect, they found instabilities when $f$ is decreasing. However, no instabilities are found when $f$ is increasing. Their analysis may be relevant in the stick-slip regime, but does not seem to be capable of explaining what happens in the sharkskin and wavy regimes.

The observed phenomena in the sharkskin regime suggest an instability to short wave disturbances, with eigenmodes that are essentially confined to the boundary. In recent work by the author [4], such instabilities were found for elastic solids sliding under Coulomb friction. The analysis of [4] easily carries over to Maxwell fluids, but its relevance appears doubtful. While significant effects of pressure on slip have been observed in rubber compounds [5], the effect of pressure in polymer melts seems to be controversial and in any case not very large. This makes it unlikely that the instability criterion derived in [4] would apply. In the present paper, we investigate a different possibility, namely that of memory slip. Rather than postulating that the slip velocity is a function of the shear stress, we make it depend on the history of the shear stress; specifically, we postulate a relationship of the form $\frac{d u}{d t}+\eta u=f(\tau)$. On a physical level, a relationship of this kind may arise from a competition between high stresses, which cause loss of adhesion, and a relaxation mechanism, by which adhesion is restored. The possibility of memory slip was suggested in [3], but its consequences were not fully explored. We shall see that short wave instabilities occur in this case. These instabilities are an instance of ill-posedness, since the growth rate tends to infinity as the wavelength tends to zero. In that respect, the instability is similar to Hadamard instabilities (see e.g. [6]), which have been proposed as an explanation for melt fracture in the past. The main difference is that, while Hadamard instabilities occur in the interior of the flow domain, the instability discussed here is confined to the boundary. 


\section{Ill-posedness for a Maxwell fluid with memory slip}

We consider two-dimensional flow of an upper convected Maxwell fluid with the constitutive law

$$
\frac{\partial \mathbf{T}}{\partial t}+(\mathbf{v} \cdot \nabla) \mathbf{T}-(\nabla \mathbf{v}) \mathbf{T}-\mathbf{T}(\nabla \mathbf{v})^{T}+\Lambda \mathbf{T}=\mu\left(\nabla \mathbf{v}+(\nabla \mathbf{v})^{T}\right)
$$

In components, we write

$$
\mathbf{v}=(u, v), \mathbf{T}=\left(\begin{array}{ll}
\sigma & \tau \\
\tau & \gamma
\end{array}\right)
$$

We consider perturbations of a basic shear flow in the domain $y>0$. At the boundary $y=0$, we have the slip velocity $U$, and stress components $\Sigma, \Upsilon$ and $\Gamma=0$. We linearize the equations of motion at these values. We separate variables and seek solutions proportional to $\exp (i \alpha x+\lambda t)$, where $\alpha>0$. Derivatives with respect to $y$ will be denoted by a prime. We are interested in the case where $\alpha$ is large, and we look for eigenmodes such that $\lambda+i \alpha U$ is of order $\sqrt{\alpha}$, stresses are of order $\sqrt{\alpha}$ relative to velocities, and the eigenfunction decays away from the boundary at a rate proportional to $\alpha$. To leading order in $\alpha$, the resulting linear problem is

$$
\begin{gathered}
(\lambda+i \alpha U) \sigma-2(\Sigma+\mu) i \alpha u-2 \Upsilon u^{\prime}=0 \\
(\lambda+i \alpha U) \tau-(\Sigma+\mu) i \alpha v-\mu u^{\prime}=0 \\
(\lambda+i \alpha U) \gamma-2 \Upsilon i \alpha v-2 \mu v^{\prime}=0 \\
i \alpha p-i \alpha \sigma-\tau^{\prime}=0 \\
p^{\prime}-i \alpha \tau-\gamma^{\prime}=0 \\
i \alpha u+v^{\prime}=0 .
\end{gathered}
$$

We seek solutions of (3) which decay exponentially for $y>0$ and satisfy the boundary conditions

$$
\begin{gathered}
v=0 \\
(\lambda+i \alpha U) u=\nu \tau .
\end{gathered}
$$

If we find solutions of this kind for which $\operatorname{Re} \lambda>0$, then we have blow-up of shortwave disturbances at the boundary and the problem is ill-posed. The second equation in (4) is the linearization of the memory slip law discussed in the introduction.

The most economic way to solve (3) and (4) is by introducing the streamfunction and vorticity (see [6]). Let $u=\psi^{\prime}, v=-i \alpha \psi$ and $\zeta=u^{\prime}-i \alpha v=\psi^{\prime \prime}-\alpha^{2} \psi$. From (3) we obtain

$$
\alpha^{2}(\Sigma+\mu) \zeta-2 i \alpha \Upsilon \zeta^{\prime}-\mu \zeta^{\prime \prime}=0 .
$$

Equation (5) admits solutions $\exp (-\beta y)$, where

$$
\alpha^{2}(\Sigma+\mu)+2 i \alpha \beta \Upsilon-\mu \beta^{2}=0, \text { and hence }
$$




$$
\beta=\frac{i \alpha \Upsilon \pm \sqrt{-\alpha^{2} \Upsilon^{2}+\alpha^{2} \mu(\mu+\Sigma)}}{\mu}
$$

We note that for the upper convected Maxwell model, we always have $(\mu+\Sigma) \mu-\Upsilon^{2}>0$. One of the two values given by (6) has positive and one has negative real part. Since we want solutions which decay for $y>0$, we must choose the value with positive real part. Henceforth $\beta$ denotes this value.

The vorticity has the form $\zeta=\zeta_{0} \exp (-\beta y)$ and the streamfunction is readily obtained as

$$
\psi=\frac{\zeta_{0}}{\beta^{2}-\alpha^{2}} e^{-\beta y}+\psi_{0} e^{-\alpha y}
$$

The first equation in (4) yields

$$
\psi(0)=0, \text { and hence } \psi_{0}=-\frac{\zeta_{0}}{\beta^{2}-\alpha^{2}} .
$$

At the boundary $y=0$, we can now compute the derivatives of the velocities,

$$
u=\psi^{\prime}=(\beta-\alpha) \psi_{0}, v=0, u^{\prime}=\psi^{\prime \prime}=\left(\alpha^{2}-\beta^{2}\right) \psi_{0}, v^{\prime}=-i \alpha \psi^{\prime}=i \alpha(\alpha-\beta) \psi_{0},
$$

and the shear stress,

$$
\tau=\frac{1}{\lambda+i \alpha U}\left((\Sigma+\mu) i \alpha v+\mu u^{\prime}\right)=\frac{\mu}{\lambda+i \alpha U}\left(\alpha^{2}-\beta^{2}\right) \psi_{0},
$$

We insert these values into the second boundary condition (4) and multiply by the factor $(\lambda+i \alpha U) /\left((\beta-\alpha) \psi_{0}\right)$. This yields

$$
(\lambda+i \alpha U)^{2}=-\nu \mu(\alpha+\beta) .
$$

Since $\beta$ as given by equation (6) has non-vanishing imaginary part, one of the roots of (11) has positive real part. Since $\beta$ is proportional to $\alpha$, the real part of $\lambda$, i.e. the growth rate, turns out proportional to $\sqrt{\alpha}$.

\section{Acknowledgement}

This research was completed while the author was visiting the Institute for Mathematics and its Applications at the University of Minnesota. Financial support from the IMA and from the National Science Foundation under Grant No. DMS-8796241 is gratefully acknowledged.

\section{References}

[1] A.V. Ramamurthy, J. Rheology 30 (1986), 337

[2] D.S. Kalika and M.M. Denn, J. Rheology 31 (1987), 815

[3] J.R.A. Pearson and C.J.S. Petrie, in: R.E. Wetton and R.W. Whorlow (eds.), Polymer Systems, Deformation and Flow, Macmillan 1968

[4] M. Renardy, Ill-posedness at the boundary for elastic solids sliding under Coulomb friction, submitted to J. Elasticity

[5] R. Brzoskowski, S.A. Montes, J.L. White and N. Nakajima, JNNFM 31 (1989), 43

[6] D.D. Joseph, M. Renardy and J.C. Saut, Arch. Rat. Mech. Anal. 87 (1985), 213 
Lucas Hsu, Niky Kamran and Peter J. Olver, Equivalence of Higher Order Lagrangians II. The Cartan Form for Particle Lagrangians

D.J. Kaup and Peter J. Olver, Quantization of BiHamiltonian Systems

Metin Arik, Fahrünisa Neyzi, Yavuz Nutku, Peter J. Olver and John M. Verosky Multi-Hamiltonian Structure of the Born-Infeld Equation

David H. Wagner, Detonation Waves and Deflagration Waves in the One Dimensional ZND Model for High Mach Number Combustion

Jerrold R. Griggs and Daniel J. Kleitman, Minimum Cutsets for an Element of a Boolean Lattice

Dieter Jungnickel, On Affine Difference Sets

Pierre Leroux, Reduced Matrices and q-log Concavity Properties of q-Stirling Numbers

A. Narain and Y. Kizilyalli, The Flow of Pure Vapor Undergoing Film Condensation Between Parallel Plates

Donald A. French, On the Convergence of Finite Element Approximations of a Relaxed Variational Problem

Yisong Yang, Computation, Dimensionality, and Zero Dissipation Limit of the Ginzburg-Landau Wave Equation

Jürgen Sprekels, One-Dimensional Thermomechanical Phase Transitions with Non-Convex Potentials of Ginzburg-Landau Type

Yisong Yang, A Note On Nonabelian Vortices

Yisong Yang, On the Abelian Higgs Models with Sources

Chjan. C. Lim, Existence of Kam Tori in the Phase Space of Vortex Systems

John Weiss, Bäcklund Transformations and the Painlevé Property

$\mathrm{Pu}$ Fu-cho and D.H. Sattinger, The Yang-Baxter Equation for Integrable Systems

E. Bruce Pitman and David G. Schaeffer, Instability and Ill-Posedness in Granular Flow

Brian A. Coomes, Polynomial Flows on $\mathbb{C}^{n *}$

Bernardo Cockburn, Suchung Hou and Chi-Wang Shu, The Runge-Kutta Local Projection Discontinuous Galerkin Finite Element Method for Conservation Laws IV: The Multidimensional Case

Peter J. Olver, Invariant Theory, Equivalence Problems, and the Calculus of Variations

Daniel D. Joseph and Thomas S. Lundgren with an appendix by R. Jackson and D.A. Saville, Ensemble Averaged and Mixture Theory Equations

P. Singh, Ph. Caussignac, A. Fortes, D.D. Joseph and T. Lundgren, Stability of Periodic Arrays of Cylinders Across the Stream by Direct Simulation

Daniel D. Joseph, Generalization of the Foscolo-Gibilaro Analysis of Dynamic Waves

A. Narain and D.D. Joseph, Note on the Balance of Energy at a Phase Change Interface

Daniel D. Joseph, Remarks on inertial radii, persistent normal stresses, secondary motions, and non-elastic extensional viscosities

D. D. Joseph, Mathematical Problems Associated with the Elasticity of Liquids

Henry C. Simpson and Scott J. Spector, Some Necessary Conditions at an Internal Boundary for Minimizers in Finite Elasticity

Peter Gritzmann and Victor Klee, On the 0-1 Maximization of Positive Definite Quadratic Forms

Fu-Cho Pu and D.H. Sattinger, The Yang-Baxter Equations and Differential Identities

Avner Friedman and Fernando Reitich, A Hyperbolic Inverse Problem Arising in the Evolution of Combustion Aerosol

E.G. Kalnins, Raphael D. Levine and Willard Miller, Jr., Conformal Symmetries and Generalized Recurrences for Heat and Schrödinger Equations in One Spatial Dimension

Wang Jinghua and Gerald Warnecke, On Entropy Consistency of Large Time Step Godunov and Glimm Schemes

C. Guillopé and J.C. Saut, Existence Results for the Flow of Viscoelastic Fluids with a Differential Constitutive Law

H.L. Bodlaender, P. Gritzmann, V. Klee and J. Van Leeuwen Computational Complexity of Norm-Maximization

Li Ta-tsien (Li Da-qian) and Yu Xin, Life-Span of Classical Solutions to Fully Nonlinear Wave Equations

Jong-Shenq Guo, A Variational Inequality Associated with a Lubrication Problem

Jong-Shenq Guo, On the Semilinear Elliptic Equation $\Delta w-\frac{1}{2} y \cdot \nabla w+\lambda w-w^{-\beta}=0$ in $R^{n}$.

Andrew E. Yagle, Inversion of the Bloch transform in magnetic resonance imaging using asymmetric two-component inverse scattering 


\section{Recent IMA Preprints (Continued)}

Title

Bei Hu, A Fiber Tapering Problem

Peter J. Olver, Canonical Variables for BiHamiltonian Systems

Michael Renardy, A Well-Posed Boundary Value Problem for Supercritical Flow of Viscoelastic Fluids of Maxwell Type

Michael Renardy, Ill-Posedness Resulting from Slip As a Possible Explanation of Melt Fracture

Michael Renardy, Compatibility Conditions at Corners Between Walls and Inflow Boundaries for Fluids of Maxwell Type

Rolf Rees, The Spectrum of Restricted Resolvable Designs with $r=2$

D. Lewis and J.C. Simo, Nonlinear stability of rotating pseudo-rigid bodies

Robert Hardt and David Kinderlehrer, Variational Principles with Linear Growth

San Yih Lin and Yisong Yang, Computation of Superconductivity in Thin Films

A. Narain, Pressure Driven Flow of Pure Vapor Undergoing Laminar Film Condensation Between Parallel Plates

P.J. Vassiliou, On Local Equivalence for Vector Field Systems

Brian A. Coomes, On Conditions Sufficient for Injectivity of Maps

Yanchun Zhao, A Class of Global Smooth Solutions of the One Dimensional Gas Dynamics System

H. Holden, L. Holden and N.H. Risebro, Some Qualitative Properties of $2 \times 2$ Systems of Conservation Laws of Mixed Type

M. Slemrod, Dynamics of Measured Valued Solutions to a Backward-Forward Heat Equation

Avner Friedman and Jürgen Sprekels, Steady States of Austenitic-Martensitic-Domains in the Ginzburg-Landau Theory of Shape Memory Alloys

Avner Friedman and Bei Hu, Degenerate Hamilton-Jacobi-Bellman Equations in a Bounded Domain

E.G. Kalnins, Willard Miller, Jr., and M.V. Tratnik, Families of Orthogonal and Biorthogonal Polynomials on the N-Sphere

Heinrich Freistühler, On Compact Linear Degeneracy

Matthew Witten, Quantifying the Concepts of Rate and Acceleration/Deceleration of Aging

J.P. Albert and J.L. Bona, Total Positivity and the Stability of Internal Waves in Stratified Fluids of Finite Depth

Brian Coomes and Victor Zurkowski, Linearization of Polynomial Flows and Spectra of Derivations

Yuriko Renardy, A Couette-Poiseuille Flow of Two Fluids in a Channel

Michael Renardy, Short wave instabilities resulting from memory slip

Daniel D. Joseph and Michael Renardy, Stokes' first problem for linear viscoelastic fluids with finite memory

Xiaxi Ding, Superlinear Conservation Law with Viscosity

J.L. Ericksen, Liquid Crystals with Variable Degree of Orientation

F. Robert Ore, Jr. and Xinfu Chen, Electro-Optic Modulation in an Arbitrary Cross-Section Waveguide

M.V. Tratnik, Multivariable biorthogonal continuous-discrete Wilson and Racah polynomials

Yisong Yang, Existence of Solutions for a Generalized Yang-Mills Theory

Peter Gritzmann, Laurent Habsieger and Victor Klee, Good and Bad Radii of Convex Polygons

Martin Golubitsky, Martin Krupa and Chjan. C. Lim, Time-Reversibility and Particle Sedimentation

G. Yin, Recent Progress in Parallel Stochastic Approximations

G. Yin, On H-Valued SA: Finite Dimensional Approximations

Chien-Cheng Chang, Accurate Evaluation of the Effect of Diffusion and Conductivity in Certain Equations

Chien-Cheng Chang and Ruey-Ling Chern, The Effect of Viscous Diffusion in Discrete Vortex Dynamics for Slightly Viscous Flows

Li Ta-Tsien (Li Da-qian) and Zhao Yan-Chun, Global Existence of Classical Solutions to the Typical Free Boundary Problem for General Quasilinear Hyperbolic Systems and its Applications

Thierry Cazenave and Fred B. Weissler, The Structure of Solutions to the Pseudo-Conformally Invariant Nonlinear Schrödinger Equation

Marshall Slemrod and Athanasios E. Tzavaras, A Limiting Viscosity Approach for the Riemann Problem in Isentropic Gas Dynamics

Richard D. James and Scott J. Spector, The Formation of Filamentary Voids in Solids

P.J. Vassiliou, On the Geometry of Semi-Linear Hyperbolic Partial Differential Equations in the Plane Integrable by the Method of Darboux

Jerome V. Moloney and Alan C. Newell, Nonlinear Optics

Keti Tenenblat, A Note on Solutions for the Intrinsic Generalized Wave and Sine-Gordon Equations

P. Szmolyan, Heteroclinic Orbits in Singularly Perturbed Differential Equations

Wenxiong Liu, A Parabolic System Arising In Film Develnpment 